\title{
A discontinuous residual-free bubble method for advection-diffusion problems
}

\author{
Giancarlo Sangalli \\ I.M.A.T.I.-C.N.R. \\ Via Ferrata 1, 27100 Pavia, Italy \\ e-mail: sangalli@imati.cnr.it
}

\begin{abstract}
A discontinuous finite element method is presented for solving the linear advectiondiffusion equation, based on the Residual-Free Bubble (RFB) finite-element formulation. After the macro-scales (usual piecewise-polynomials elements) are separated from the micro-scales (the bubble part), they are computed by a plain Galerkin formulation, while the bubble part is approximated by a discontinuous Galerkin method. The advantage of this approach, as compared to other implementations of the Residual-Free Bubble formulation, is that the macro-scales are computed accurately, at least for the model problem presently considered. Numerical tests are performed on a two-dimensional model problem to confirm the validity of the proposed approach.
\end{abstract}

keywords: advection-diffusion, finite element method, stabilization, SUPG, residual-free bubbles, multiscale.

AMS Subject Classification: 65F10, 65N30, 65N55

\section{Introduction}

In this paper, we present a numerical procedure based on the Residual-Free Bubble (RFB) Finite Element Method (FEM) for solving of the linear advection-diffusion equation. This simple model problem encompasses one of the difficulties encountered in the numerical simulation of fluid flow (e.g., [21,22]). It is well known that classical numerical methods - such as the central finite difference method or the plain Galerkin FEM - are inadequate when the diffusive term is small compared to the advective term. Typically in our model problem, but also in real fluid flow simulation, unphysical oscillations pollute the numerical solution in the whole domain, while the exact solution only shows boundary or internal layers.

To overcome this difficulty, so-called stabilized methods have been developed. In the framework of finite element methods, a simple modification consists of adding a suitable amount of artificial diffusion. This idea was developed by Hughes and collaborators in the eighties $[12,18,19]$. Their Streamline-Upwind Petrov-Galerkin (SUPG) method adds diffusion only in the streamline direction, that is, in the direction of the advection field, while preserving the 
consistency of the variational formulation. The SUPG technique performs better than the naive artificial diffusion technique, as shown by the theoretical analysis [12], and also confirmed by numerical tests (e.g. [22]). SUPG and its variants (such as the Galerkin Least-Squares method) have become the most popular numerical methods for this kind of problems among FEMs.

Despite the success of SUPG, there are areas for improvement. For example, because SUPG is not monotone, it does not preserve the positivity of the solution, which is unphysical in some applications. Another weakness of SUPG is that the amount of streamline diffusion has to be tuned depending on the problem at hand. For our simple model problem, an effective tuning of SUPG is available (see [12]), while in other cases, for example, in real-world fluid flow simulation, tuning of the method can be difficult. This difficulty has motivated the introduction of intrinsically stable methods. Examples include the Variational Multiscale method of Hughes and coworkers (see [17]), and the Residual-Free Bubbles (RFB) method of Brezzi and Russo (in [10]). These two methods are closely related, as discussed in [3]. A detailed discussion on the advantages and disadvantages of the methods can be found in references $[4,9,17]$.

In particular, the Residual-Free Bubble (RFB) method is based on a local enrichment of the finite element space instead of a modification of the variational formulation. The idea is to add to the usual space of piecewise polynomials, referred to as macro-scales in this paper, the so-called bubbles, representing the micro-scales. Bubbles are functions whose support remains inside the elements of the triangulation. The numerical method turns out to be intrinsically stable (see, for example, [7] and [23]), at the price of having to solve local problems in order to approximate, and possibly eliminate, the infinite bubble degrees of freedom. In one dimension, the local problems can be solved analytically, and the final numerical scheme produces nodally exact numerical solutions (see [10]). In the multi-dimensional case, one can approximate analytically the bubble effect only in particular cases; for example, in [10] the case of linear elements is considered. In more general cases, procedures for dealing with the bubble degrees of freedom have been proposed, as will be discussed in $\S 2$.

In this paper, we propose to approximate the local problems for the bubble degrees of freedom of the Residual-Free Bubble (RFB) formulation by means of a discontinuous Galerkin method. This approach has the advantage of allowing us to accurately compute the effect of the bubbles on the macro-scales when using linear or higher order elements, in the advectiondominated case. In $\S 2$, we present the Residual-Free Bubble (RFB) idea and discuss the practical implementation, also including the new proposal. In $\S 3$, we present numerical tests, and in $\S 4$ we discuss the conclusions.

\section{The RFB formulation and implementation}

We consider the linear advection-diffusion problem

$$
\mathcal{L}_{\varepsilon} u=f \quad \text { in } \Omega,
$$

subject to the homogeneous Dirichlet boundary condition, where

$$
\mathcal{L}_{\varepsilon}:=-\varepsilon \Delta+\mathbf{c} \cdot \nabla
$$

$\nabla$ denotes the gradient operator, i.e., the vector of first derivatives, and $\Delta$ denotes the Laplace operator, i.e.,

$$
\Delta:=\sum_{i} \frac{\partial^{2}}{\partial x_{i}^{2}}
$$


The unknown real-valued function $u$ is defined on the convex polygonal domain $\Omega \subset \mathbb{R}^{2}, \varepsilon$ is a strictly positive diffusivity coefficient, and $\mathbf{c}$ is the velocity field in $\Omega$. As mentioned in the introduction, this model problem encompasses some of the difficulties encountered in the numerical simulations of fluid flow (see, e.g., [22]). The variational formulation of (1) can be stated as follows: find $u \in H_{0}^{1}(\Omega)$ such that

$$
a(u, v)=\langle f, v\rangle, \quad \forall v \in H_{0}^{1}(\Omega),
$$

where

$$
a(w, v):=\varepsilon \int_{\Omega} \nabla w \cdot \nabla v d \mathbf{x}+\int_{\Omega}(\mathbf{c} \cdot \nabla w) v d \mathbf{x}
$$

and

$$
\langle f, v\rangle:=\int_{\Omega} f v d \mathbf{x}
$$

We shall assume that $f$ belongs to $L^{2}(\Omega)$, and $\operatorname{div}(\mathbf{c}) \leq 0$. This guarantees that the variational formulation of (1) is well posed (see [20]). Given a subset $\omega$ of the domain $\Omega$ (possibly the whole $\Omega$ itself), we follow the usual notation for the Lebesgue spaces $L^{p}(\omega)(1 \leq p \leq \infty)$ and Sobolev space $H^{1}(\omega)$ of functions whose partial derivatives are in $L^{2}(\omega)$, and denote by $H_{0}^{1}(\omega)$ the subspace of $H^{1}(\omega)$ of all functions vanishing on the boundary $\partial \omega$ (see [20]). Moreover, we denote by $\partial \omega^{-}, \partial \omega^{0}$ and $\partial \omega^{+}$, respectively, the inflow boundary, the characteristic boundary, and the outflow boundary,

$$
\begin{aligned}
\partial \omega^{-} & :=\{\mathbf{x} \in \partial \omega \text { such that } \mathbf{c} \cdot \mathbf{n}<0\} \\
\partial \omega^{0}: & =\{\mathbf{x} \in \partial \omega \text { such that } \mathbf{c} \cdot \mathbf{n}=0\}, \\
\partial \omega^{+} & :=\{\mathbf{x} \in \partial \omega \text { such that } \mathbf{c} \cdot \mathbf{n}>0\},
\end{aligned}
$$

where $\mathbf{n}$ is the unit outward normal vector.

We shall deal with a family of partitions $\mathcal{T}_{h}$ of the domain $\Omega$ into open triangles, satisfying the usual conditions of admissibility (any two elements have disjoint closure, a vertex in common, or share a complete edge), and shape regularity (see [14]). The diameter of an element $T$ will be denoted by $h_{T}$, and the maximum diameter of all elements in $\mathcal{T}_{h}$ will be denoted by $h$.

We also assume that $\mathbf{c}$ and $f$ are piecewise constant on the triangulation $\mathcal{T}_{h}$. Consequently, the assumption $\operatorname{div}(\mathbf{c}) \leq 0$ has to be accepted in the sense of distributions, i.e., $\mathbf{c} \cdot \mathbf{n}_{T_{1}}+\mathbf{c} \cdot \mathbf{n}_{T_{2}} \leq 0$ on the common edge $\partial T_{1} \cup \partial T_{2}$ of any two elements $T_{1}, T_{2}$ of $\mathcal{T}_{h}$, where $\mathbf{n}_{T_{i}}$ denotes the outward direction on $\partial T_{i}$. We shall focus our attention on the advection-dominated regime, where $\varepsilon$ is large compared to $h_{T}\left\|\mathbf{c}_{\mid T}\right\|$ in each element $T \in \mathcal{T}_{h}$. This is indeed the regime where standard numerical methods are inadequate (see [22]).

Consider the usual conforming finite-dimensional space of order $k \geq 1$,

$$
V_{P} \equiv V_{P}\left(\mathcal{T}_{h}, k\right):=\left\{v \in H_{0}^{1} \text { such that } v_{\mid T} \in \mathbb{P}_{k}, \forall T \in \mathcal{T}_{h}\right\}
$$

where $\mathbb{P}_{k}$ denotes the space of polynomials of degree $k$. The Streamline-Upwind Petrov-Galerkin (SUPG) method can be stated as follows: find $u_{P}^{S U P G} \in V_{P}$, such that

$$
a\left(u_{P}^{S U P G}, v_{P}\right)+\sum_{T \in \mathcal{T}_{h}} \tau_{T} \int_{T} \mathcal{L}_{\varepsilon} u_{P}^{S U P G} \mathbf{c} \cdot \nabla v_{P}=\left\langle f, v_{P}\right\rangle+\sum_{T \in \mathcal{T}_{h}} \tau_{T} \int_{T} f \mathbf{c} \cdot \nabla v_{P}, \forall v_{P} \in V_{P}
$$


where $\tau_{T}$ is the artificial streamline diffusion parameter [12],

$$
\tau_{T}:=\frac{h_{T}}{2\|\mathbf{c}\|}, \quad \text { in } T \in \mathcal{T}_{h}
$$

The Residual-Free Bubble (RFB) approach was proposed by Brezzi and Russo [10], inspired by a different philosophy: taking the variational formulation of (1) without modification, the numerical solution is found in the enriched space $V_{E}$ of functions that are piecewise polynomials on the boundaries of the elements,

$$
V_{E} \equiv V_{E}\left(\mathcal{T}_{h}, k\right):=\left\{v \in H_{0}^{1} \text { such that } v_{\mid \partial T} \in \mathbb{P}_{k}, \forall T \in \mathcal{T}_{h}\right\}
$$

The Residual-Free Bubble (RFB) formulation is stated as follow: find $u_{E}^{R F B} \in V_{E}$, such that

$$
a\left(u_{E}^{R F B}, v_{E}\right)=\left\langle f, v_{E}\right\rangle, \quad \forall v_{E} \in V_{E} .
$$

An error analysis for the Residual-Free Bubble (RFB) method was presented in references $[8,23]$. Note that the stabilizing mechanism is intrinsically contained in the enrichment of the space. Contrary to the Streamline-Upwind Petrov-Galerkin (SUPG) formulation, there are no free parameters. Because of the presence of the bubbles, (8) is an infinite-dimensional variational formulation, and cannot be coded into a numerical algorithm. To develop an algorithm, we need to approximate (8) with a finite number of degrees of freedom. The aim of this paper is to propose and justify an original finite-dimensional approximation of (8) based on a non-conforming (discontinuous) approximation of $V_{E}$.

We shall denote by $\mathcal{L}_{0}:=\mathbf{c} \cdot \nabla(\cdot)$ the purely hyperbolic operator, which is the formal limit of $\mathcal{L}_{\varepsilon}$ when $\varepsilon \rightarrow 0 ; \mathcal{L}_{\varepsilon}^{*}:=-\varepsilon \Delta(\cdot)-\operatorname{div}(\mathbf{c}(\cdot))$ and $\mathcal{L}_{0}^{*}:=-\operatorname{div}(\mathbf{c}(\cdot))$ denote the adjoint of $\mathcal{L}_{\varepsilon}$ and $\mathcal{L}_{0}$, respectively. In particular, if we restrict our attention to (the interior of) an element $T \in \mathcal{T}_{h}$, where $\mathbf{c}$ is assumed to be constant, then $\mathcal{L}_{\varepsilon}^{*}:=-\varepsilon \Delta(\cdot)-\mathbf{c} \cdot \nabla(\cdot)$ and $\mathcal{L}_{0}^{*}:=\mathbf{c} \cdot \nabla(\cdot)$.

The key idea in developing an algorithm from (8) is the distinction between macro-scales, which are represented by piecewise polynomials, and micro-scales, or bubbles, which reside inside the elements. We therefore assume that any $v_{E} \in V_{E}$ admits a unique decomposition in

$$
v_{E}=v_{P}+v_{B} \text {, with } v_{P} \in V_{P}, v_{B} \in V_{B},
$$

where the bubble space is

$$
V_{B} \equiv V_{B}\left(\mathcal{T}_{h}\right):=\left\{v_{B}: v_{B \mid T} \in H_{0}^{1}(T), \forall T \in \mathcal{T}_{h}\right\}
$$

Note that, in order to have a unique splitting (9), namely $V_{E}=V_{P} \oplus V_{B}$, we must restrict the order of polynomials to $1 \leq k \leq 2$. Indeed, in a triangular element, we can have bubbles which are polynomials of order 3 or higher; an example is the product of the usual barycentric coordinates, i.e., of the distances from the edges of the element. Therefore, as usual, we split $u_{E}^{R F B}=u_{P}^{R F B}+u_{B}^{R F B}$, where $u_{P}^{R F B} \in V_{P}$ and $u_{B}^{R F B} \in V_{B}$, and test (8) using $v_{P} \in V_{P}, v_{B} \in V_{B}$, yielding

$$
\begin{array}{ll}
a\left(u_{P}^{R F B}, v_{P}\right)+a\left(u_{B}^{R F B}, v_{P}\right)=\left\langle f, v_{P}\right\rangle, & \forall v_{P} \in V_{P} \\
a\left(u_{P}^{R F B}, v_{B}\right)+a\left(u_{B}^{R F B}, v_{B}\right)=\left\langle f, v_{B}\right\rangle, & \forall v_{B} \in V_{B} .
\end{array}
$$


Equation (12) gives $u_{B}^{R F B}$ from $u_{P}^{R F B}$ and $f$ : actually, $u_{B}^{R F B}$ solves in each element, $T$, the boundary-value problem

$$
\left\{\begin{aligned}
\mathcal{L}_{\varepsilon} u_{B}^{R F B} & =f-\mathcal{L}_{\varepsilon} u_{P}^{R F B} & & \text { in } T, \\
u_{B}^{R F B} & =0 & & \text { on } \partial T .
\end{aligned}\right.
$$

Substituting $u_{B}^{R F B}$ in (11), we obtain a closed-form problem for $u_{P}^{R F B}$. If $M(w)$ and $F(f)$ are , respectively, the solutions in each element, $T$, of the problems

$$
\left\{\begin{aligned}
\mathcal{L}_{\varepsilon} M(w) & =-\mathcal{L}_{\varepsilon} w & & \text { in } T \\
M(w) & =0 & & \text { on } \partial T,
\end{aligned}\right.
$$

and,

$$
\left\{\begin{aligned}
\mathcal{L}_{\varepsilon} F(f)=f & & \text { in } T \\
F(f)=0 & & \text { on } \partial T,
\end{aligned}\right.
$$

then the final variational formulation for $u_{P}^{R F B}$, after integrating by parts, is

$$
a\left(u_{P}^{R F B}, v_{P}\right)+\sum_{T \in \mathcal{T}_{h}} \int_{T} M\left(u_{P}^{R F B}\right) \mathcal{L}_{\varepsilon}^{*} v_{P}=\left\langle f, v_{P}\right\rangle-\sum_{T \in \mathcal{T}_{h}} \int_{T} F(f) \mathcal{L}_{\varepsilon}^{*} v_{P}, \quad \forall v_{P} \in V_{P} .
$$

Although this is a finite-dimensional problem, it contains the terms $\int_{T} M\left(u_{P}^{R F B}\right) \mathcal{L}_{\varepsilon}^{*} v_{P}$ and $\int_{T} F(f) \mathcal{L}_{\varepsilon}^{*} v_{P}$, which implicitly involve the solution of local infinite-dimensional problems. As proposed in [10], we can use the approximations

$$
\begin{aligned}
\sum_{T \in \mathcal{T}_{h}} \int_{T} M\left(u_{P}^{R F B}\right) \mathcal{L}_{\varepsilon}^{*} v_{P} & \approx \sum_{T \in \mathcal{T}_{h}} \int_{T} \widetilde{M}\left(u_{P}^{R F B}\right) \mathcal{L}_{0}^{*} v_{P}, \\
\sum_{T \in \mathcal{T}_{h}} \int_{T} F(f) \mathcal{L}_{\varepsilon}^{*} v_{P} & \approx \sum_{T \in \mathcal{T}_{h}} \int_{T} \widetilde{F}(f) \mathcal{L}_{0}^{*} v_{P},
\end{aligned}
$$

where, in each element $T, \widetilde{M}(w)$ and $\widetilde{F}(f)$ are given, respectively, by

$$
\left\{\begin{aligned}
\mathcal{L}_{0} \widetilde{M}(w) & =-\mathcal{L}_{0} w & & \text { in } T \\
\widetilde{M}(w) & =0 & & \text { on } \partial T^{-},
\end{aligned}\right.
$$

and

$$
\left\{\begin{aligned}
\mathcal{L}_{0} \widetilde{F}(f) & =f & & \text { in } T \\
\widetilde{F}(f) & =0 & & \text { on } \partial T^{-} .
\end{aligned}\right.
$$

Roughly speaking, (16) are justified as $\varepsilon \ll h_{T}\left\|\mathbf{c}_{\mid T}\right\|$, and so $\widetilde{M}(w)$ and $\widetilde{F}(f)$ are accurate approximations of $M(w)$ and $F(f)$ in $L^{2}(T)$, for all element $T \in \mathcal{T}_{h}$. Indeed, by virtue of the asymptotic expansion techniques of [22], one may think of $M(w)$ (resp. $F(f)$ ) as the sum of $\widetilde{M}(w)$ (resp. $\widetilde{F}(f)$ ) and a negligible boundary layer. For $k=1$, corresponding to linear elements being used as macro-scales, the solutions of (17) and (18) can be easily evaluated analytically, as shown in [10]. From (16), we can define an approximated Static Condensation of the bubble degrees of freedom, and search for $u_{P}^{S C}$ such that

$$
a\left(u_{P}^{S C}, v_{P}\right)+\sum_{T \in \mathcal{T}_{h}} \int_{T} \widetilde{M}\left(u_{P}^{S C}\right) \mathcal{L}_{0}^{*} v_{P}=\left\langle f, v_{P}\right\rangle-\sum_{T \in \mathcal{T}_{h}} \int_{T} \widetilde{F}(f) \mathcal{L}_{0}^{*} v_{P}, \quad \forall v_{P} \in V_{P} .
$$


Because of (16), we may expect

$$
u_{P}^{S C} \approx u_{P}^{R F B} .
$$

For $k=1$, both (19) and the original scheme (15)) reduce to the Streamline-Upwind PetrovGalerkin (SUPG) scheme (5), with a special choice of the streamline diffusion $\tau_{T}$. the choice $k=2$ leads to a different scheme; we refer to [10] or [1] for a more detailed analysis.

Instead of computing by hand the effect of the micro-scales on the macro-scales, a different approach involves using a suitable numerical method for the approximation of the micro-scales, namely for solving (12). Because (12) gives local problems in each element $T$ with the same structure and difficulties of the original problem (1), we are led to use an ad hoc method. We shall consider here three typical approaches:

1. The Sub-grid Viscosity (SV) method of Brezzi et al. and Guermond in [5,16], or a similar two-level method of Franca et al. in [15], which involves using an artificial diffusion or a Streamline-Upwind Petrov-Galerkin (SUPG) method for (12) on a quasi-uniform sub-grid mesh $\mathcal{T}_{h}^{S V}$ in each element $T$.

2. The Pseudo Bubble (PB) method of Brezzi et al. in [6], which involves using a standard Galerkin scheme for (12) on a suitable distorted mesh $\mathcal{T}_{h}^{P B}$ in each element $T$.

3. A new method dealing with (17)-(18) instead of (13)-(14), coined the Discontinuous Bubble (DB) method. In this formulation we solve (17)-(18) by a standard Galerkin method, but the boundary conditions (on $\partial T^{-}$) are weakly imposed through the variational formulation. Specifically, we look for a discontinuous $k$-order polynomial $u_{D}^{D B}$ which approximates $u_{B}^{R F B}$ on the mesh $\mathcal{T}_{h}^{D B} \equiv \mathcal{T}_{h}$ which leads to a non-conforming approximation of $V_{B}$, due to the discontinuities across the element boundaries.

The different meshes used in these three approaches are shown in Figure 1.

Details of the first two methodologies can be found in the references; A difficulty encountered in these approaches is that one has to tune the stabilized method for solving local problems (for example, by choosing the amount of artificial diffusion in the sub-grid viscosity case or the shape of the sub-grid mesh in the pseudo residual-free bubble case) in order to accurately compute the RFB approximation, $u_{E}^{R F B}$ or, at least, the macro-scale degrees of freedom, $u_{P}^{R F B}$. So, in a sense, the original difficulty of tuning a numerical method which depends on some parameters remains.

The proposed approach (DG) allows the accurate computation of the macro-scales $u_{P}^{R F B}$, in advection-dominated regime. We present now the idea in detail. Define the space of discontinuous piecewise polynomial functions,

$$
V_{D} \equiv V_{D}\left(\mathcal{T}_{h}, k\right):=\left\{v \in L^{2}(\Omega): v_{\mid T} \in \mathbb{P}_{k}, \forall T \in \mathcal{T}_{h}\right\}
$$

and introduce $M_{D}(w) \in V_{D}$ and $F_{D}(w) \in V_{D}$, which are the discretizations of $\widetilde{M}(w)$ and $\widetilde{F}(f)$, satisfying

$$
\int_{T} \mathbf{c} \cdot \nabla M_{D}(w) v_{D}-\int_{\partial T^{-}} M_{D}(w) v_{D} \mathbf{c} \cdot \mathbf{n}=-\int_{T} \mathbf{c} \cdot \nabla w v_{D}, \quad \forall v_{D} \in V_{D},
$$

and

$$
\int_{T} \mathbf{c} \cdot \nabla F_{D}(w) v_{D}-\int_{\partial T^{-}} F_{D}(w) v_{D} \mathbf{c} \cdot \mathbf{n}=\int_{T} f v_{D}, \quad \forall v_{D} \in V_{D}
$$



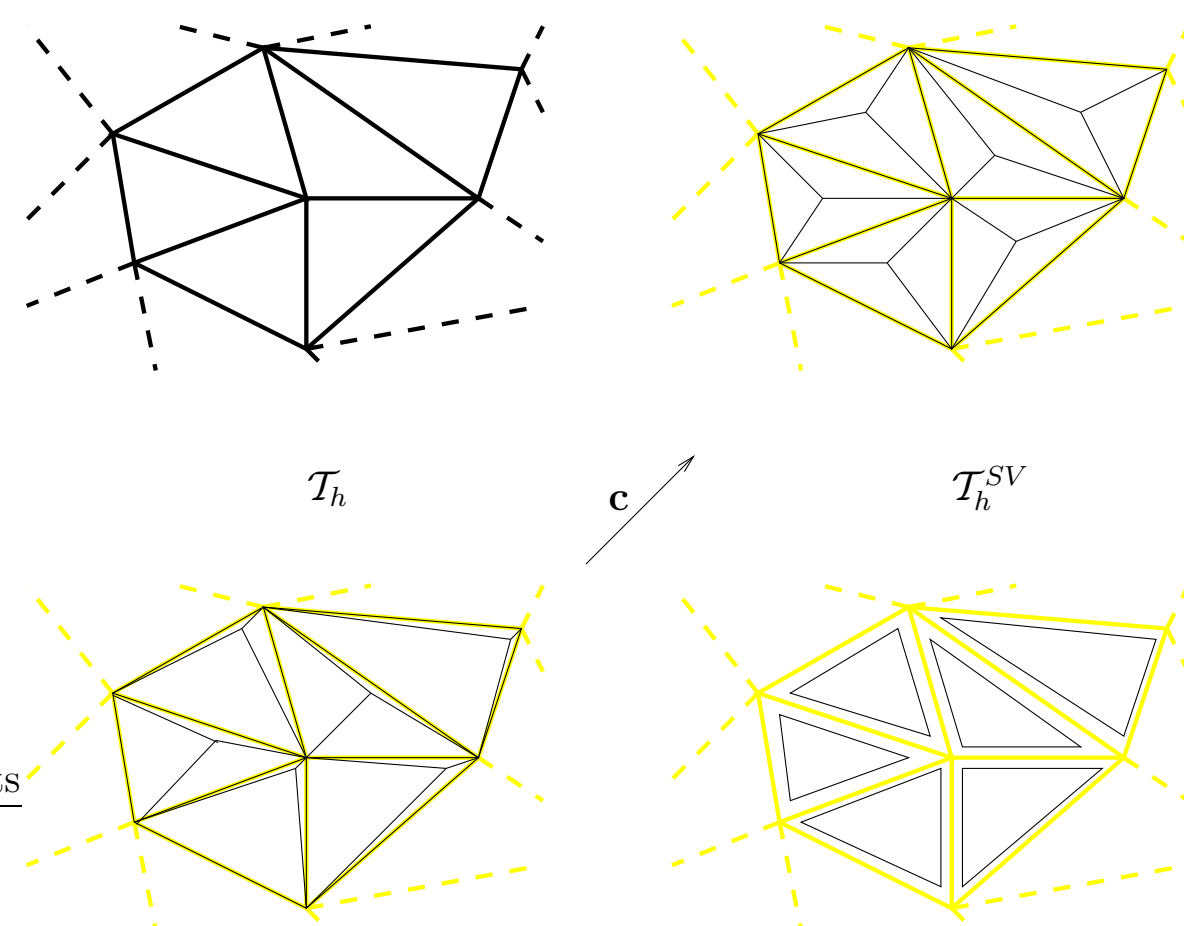

$$
\mathcal{T}_{h}^{S V}
$$

$$
\mathcal{T}_{h}^{P B}
$$

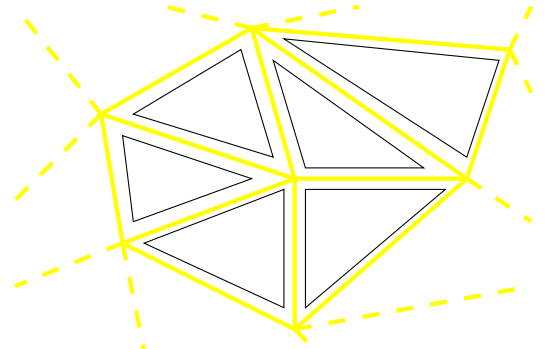

$\mathcal{T}_{h}^{D B}$

Figure 1: Examples of meshes involved in the approximation of macro and micro-scales.

Thus we define $u_{P}^{D B} \in V_{P}$ by

$$
a\left(u_{P}^{D B}, v_{P}\right)+\sum_{T \in \mathcal{T}_{h}} \int_{T} M_{D}\left(u_{P}^{D B}\right) \mathcal{L}_{0}^{*} v_{P}=\left\langle f, v_{P}\right\rangle-\sum_{T \in \mathcal{T}_{h}} \int_{T} F_{D}(f) \mathcal{L}_{0}^{*} v_{P}, \quad \forall v_{P} \in V_{P}
$$

and, since the micro-scales are approximated by $u_{D}^{D B}:=M_{D}\left(u_{P}^{D B}\right)+F_{D}(f)$, we define $u^{D B}:=$ $u_{P}^{D B}+u_{D}^{D B}$.

Concerning the implementation, we recall that (22) and (23) describe local operators which we can invert at the element level; in other words, we can compute the local matrices which represent $M_{D}(\cdot)$ and $F_{D}(\cdot)$ at the first stage, and then use them in assembling the linear system for $(24)$.

As mentioned earlier, we want to show that $u_{P}^{D B}$, given in (24), is equal to $u_{P}^{S C}$, whence our procedure is accurate in computing the macro-scales $u_{P}^{R F B}$, thanks to (20). Indeed, the formulations (19) and (24) are equivalent, because, for any $v_{P} \in V_{P}$, we have

$$
\begin{array}{r}
\sum_{T \in \mathcal{T}_{h}} \int_{T} M_{D}\left(u_{P}^{D B}\right) \mathcal{L}_{0}^{*} v_{P}=\sum_{T \in \mathcal{T}_{h}} \int_{T} \widetilde{M}\left(u_{P}^{D B}\right) \mathcal{L}_{0}^{*} v_{P} \\
\sum_{T \in \mathcal{T}_{h}} \int_{T} F_{D}(f) \mathcal{L}_{0}^{*} v_{P}=\sum_{T \in \mathcal{T}_{h}} \int_{T} \widetilde{F}(f) \mathcal{L}_{0}^{*} v_{P},
\end{array}
$$

as a consequence of the following proposition. 
Proposition 1. Consider $T \in \mathcal{T}_{h}, k \geq 1$ and $\phi \in \mathbb{P}_{k-1}$; let $w \in H^{1}(T)$ such that

$$
\left\{\begin{aligned}
\mathcal{L}_{0} w=\phi & \text { in } T \\
w=0 & \text { on } \partial T^{-},
\end{aligned}\right.
$$

and let $z \in \mathbb{P}_{k}$ be the solution of

$$
\int_{T} \mathcal{L}_{0} z v-\int_{\partial T^{-}} z v \mathbf{c} \cdot \mathbf{n}=\int_{T} \phi v, \quad \forall v \in \mathbb{P}_{k} .
$$

Then

$$
\int_{T} w \mathcal{L}_{0}^{*} v=\int_{T} z \mathcal{L}_{0}^{*} v, \quad \forall v \in \mathbb{P}_{k} .
$$

Proof. The possible orientations of the element $T$ with respect to the advection field $\mathbf{c}$ are shown in Figure 2. We denote by $\mathbf{x}^{-} \equiv \mathbf{x}^{-}(\mathbf{x}, \mathbf{c})$ the inflow point corresponding to $\mathbf{x}$, namely $\mathbf{x}^{-} \in \partial T^{-}$and the direction from $\mathbf{x}^{-}$to $\mathbf{x}$ is aligned with $\mathbf{c}$; similarly we define $\mathbf{x}^{+} \equiv \mathbf{x}^{+}(\mathbf{x}, \mathbf{c})$ as the outflow point corresponding to $\mathbf{x}$.
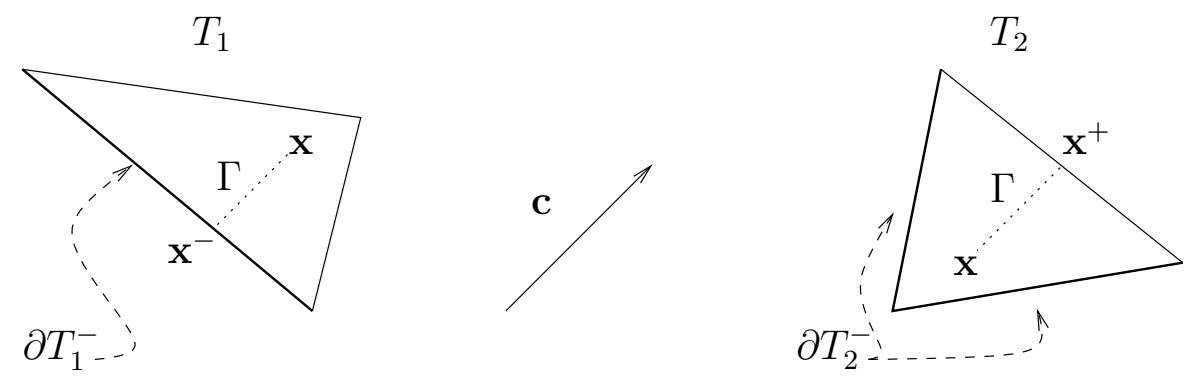

Figure 2: A triangle has either a single inflow edge, $T_{1}$, or a single outflow edge, $T_{2}$.

Consider the case of $T_{1}$ : since we have

$$
w(\mathbf{x})=|\mathbf{c}|^{-1} \int_{\mathbf{x}^{-}}^{\mathbf{x}} \phi d \Gamma,
$$

and since $\partial T_{1}^{-}$is a straight line, then $w \in \mathbb{P}_{k}$, and $w=z$, which in particular gives (27).

Consider now the element $T_{2}$, which has a single edge on the outflow boundary instead. In this case, $w \neq z$, but given $v \in \mathbb{P}_{k}$ the solution $\widetilde{v}$ of the dual problem

$$
\left\{\begin{aligned}
\mathcal{L}_{0}^{*} \widetilde{v} & =\mathcal{L}_{0}^{*} v & & \text { in } T \\
\widetilde{v} & =0 & & \text { on } \partial T^{+},
\end{aligned}\right.
$$

which is

$$
\widetilde{v}(\mathbf{x})=-|\mathbf{c}|^{-1} \int_{\mathbf{x}^{+}}^{\mathbf{x}} \mathcal{L}_{0}^{*} v d \Gamma,
$$


belongs to $\mathbb{P}_{k}$. Using $\widetilde{v}$ in (26), invoking (25) and integrating by parts we obtain

$$
\begin{aligned}
\int_{T} z \mathcal{L}_{0}^{*} \widetilde{v}+\int_{\partial T^{+}} z \widetilde{v} \mathbf{c} \cdot \mathbf{n} & =\int_{T} \mathcal{L}_{0} z \widetilde{v}+\int_{\partial T^{-}} z \widetilde{v} \mathbf{c} \cdot \mathbf{n} \\
& =\int_{T} \phi \widetilde{v} \\
& =\int_{T} \mathcal{L}_{0} w \widetilde{v} \\
& =\int_{T} w \mathcal{L}_{0}^{*} \widetilde{v}+\int_{\partial T} w \widetilde{v} \mathbf{c} \cdot \mathbf{n} \\
& =\int_{T} w \mathcal{L}_{0}^{*} \widetilde{v}+\int_{\partial T^{+}} w \widetilde{v} \mathbf{c} \cdot \mathbf{n} .
\end{aligned}
$$

Finally (28) gives (27).

\section{$3 \quad$ Numerical tests}

In this section, we test the proposed numerical method for a simple model problem. In particular, we compare results of three approaches:

- The discontinuous approximation $u^{D B}$, which contains both the macro-scales $u_{P}^{D B}$ and an approximation $u_{D}^{D B}$ of the micro-scales $u_{B}^{R F B}$ of the RFB formulation,

- The macro-scales $u_{P}^{S C}$ only; these are still obtained invoking $(24)$, since $u_{P}^{D B} \equiv u_{P}^{S C} \approx$ $u_{P}^{R F B}$, as shown above,

- The $u_{P}^{S U P G}$ approximation, given by (5).

For the sake of simplicity we restrict to linear elements (case $k=1$ ), so that $u_{P}^{D B} \equiv$ $u_{P}^{S C}$ and $u_{P}^{S U P G}$ are both given by the Streamline-Upwind Petrov-Galerkin (SUPG) variational formulation (5), the only difference being the amount of streamline diffusion $\tau_{T}$ (see [10]): we recall that for $u_{P}^{S U P G}$ we follow the notation of [12], where

$$
\tau_{T}:=\frac{h_{T}}{2\|\mathbf{c}\|}, \quad \text { in } T \in \mathcal{T}_{h} .
$$

We solve (1) in an L-shaped domain $\Omega$, where the source term $f$ and the advection field $\mathbf{c}$ are taken piecewise and discontinuous along the internal line $\Gamma_{1}$, as shown in Figure 3. Furthermore, we take $\varepsilon=10^{-5}$.

The structure of the exact solution $u$ is depicted in Figure 4; the behavior is typical of this class of problems:

- Near the outflow boundary, $\partial \Omega^{+}$, an exponential layer is present,

- Along the characteristic boundary, $\partial \Omega^{0}$, a parabolic layer is present,

- Two internal layers are present, one along $\Gamma_{1}$ (due to the discontinuity of $f$ and $\mathbf{c}$ ), and the other along $\Gamma_{2}$ (which is due to the reentrant corner in $(1 / 2,1 / 2)$ ). 


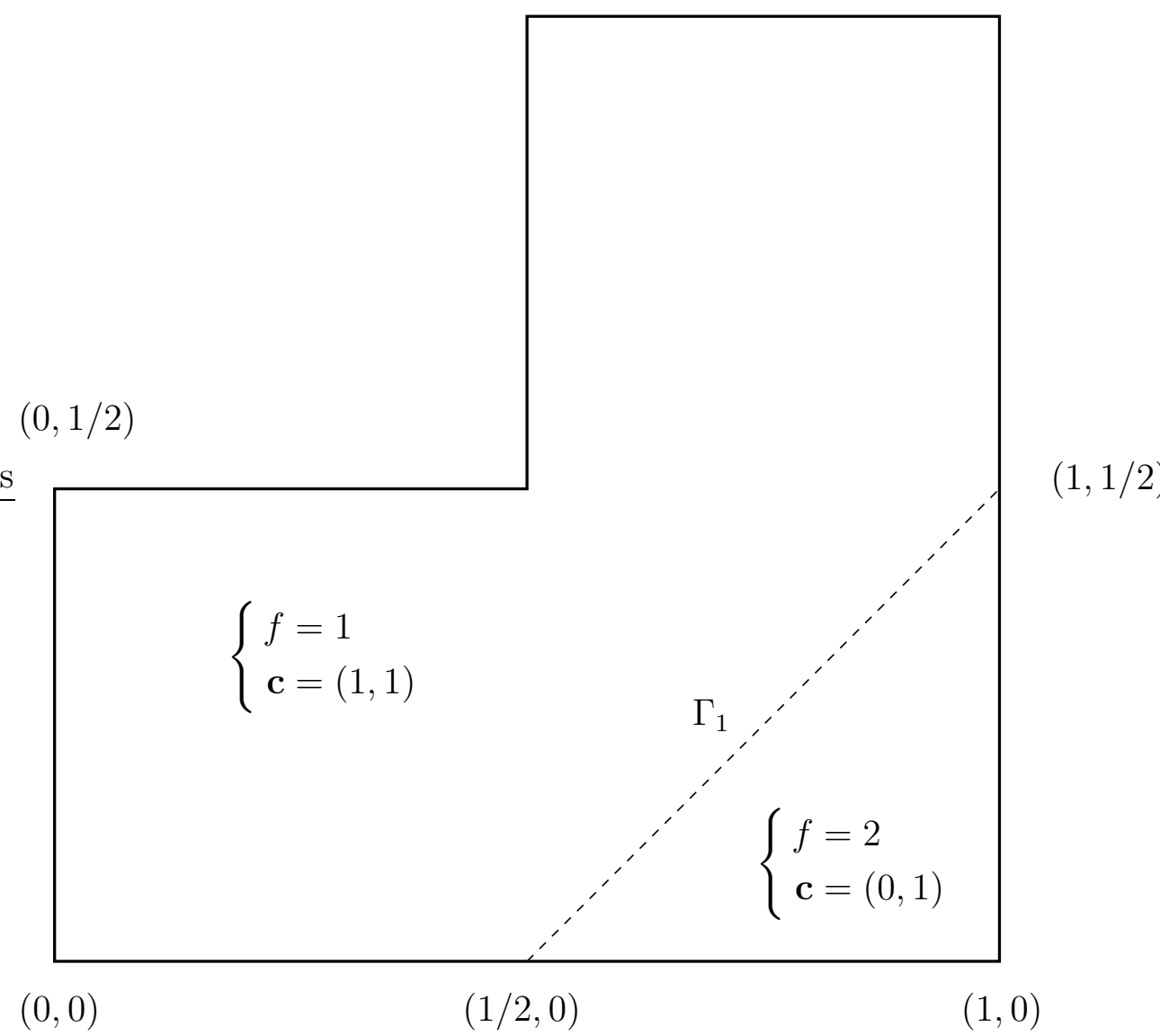

$\Omega$

Figure 3: Domain and data for the numerical test.

The domain $\Omega$ is partitioned in a quasi-uniform, non-structured, Delaunay triangulation using the Triangle routine [25]. Each triangle is required to have angles that are larger than $30^{\circ}$.

As an example, we plot the numerical solutions obtained on one mesh; we give two different points of view on each numerical solution. The first one (from NW to SE) focuses on the internal discontinuities and structures while the second one (from NE to SW) focuses on the boundary layers. We plot $u_{P}^{S U P G}$ in Figure 5(a), $u_{P}^{S C}$ in Figure 5(b) and $u^{D B}$ in Figure 5(c). In our tests, the CPU-time required for computing $u_{P}^{S U P G}, u_{P}^{S C}$ and $u^{D B}$, on a given triangulation, is almost the same.

From the plots of Figure 5(a)-5(c) we see that the numerical methods capture the structure of the exact solution, even though small spurious oscillations appear near the layers. This is not surprising, because both SUPG and RFB are non-monotone numerical methods. Moreover, it seems that our complete approximation $u^{D B}$, which contains the discontinuous approximation of the bubbles, is not better than $u_{P}^{D B} \equiv u_{P}^{S C}$. On the other hand, in Figure 6 we show the numerical error of the methods in the $L^{2}$-norm (i.e., $\left\|u-u_{P}^{S U P G}\right\|_{L^{2}},\left\|u-u_{P}^{S C}\right\|_{L^{2}}$ and 


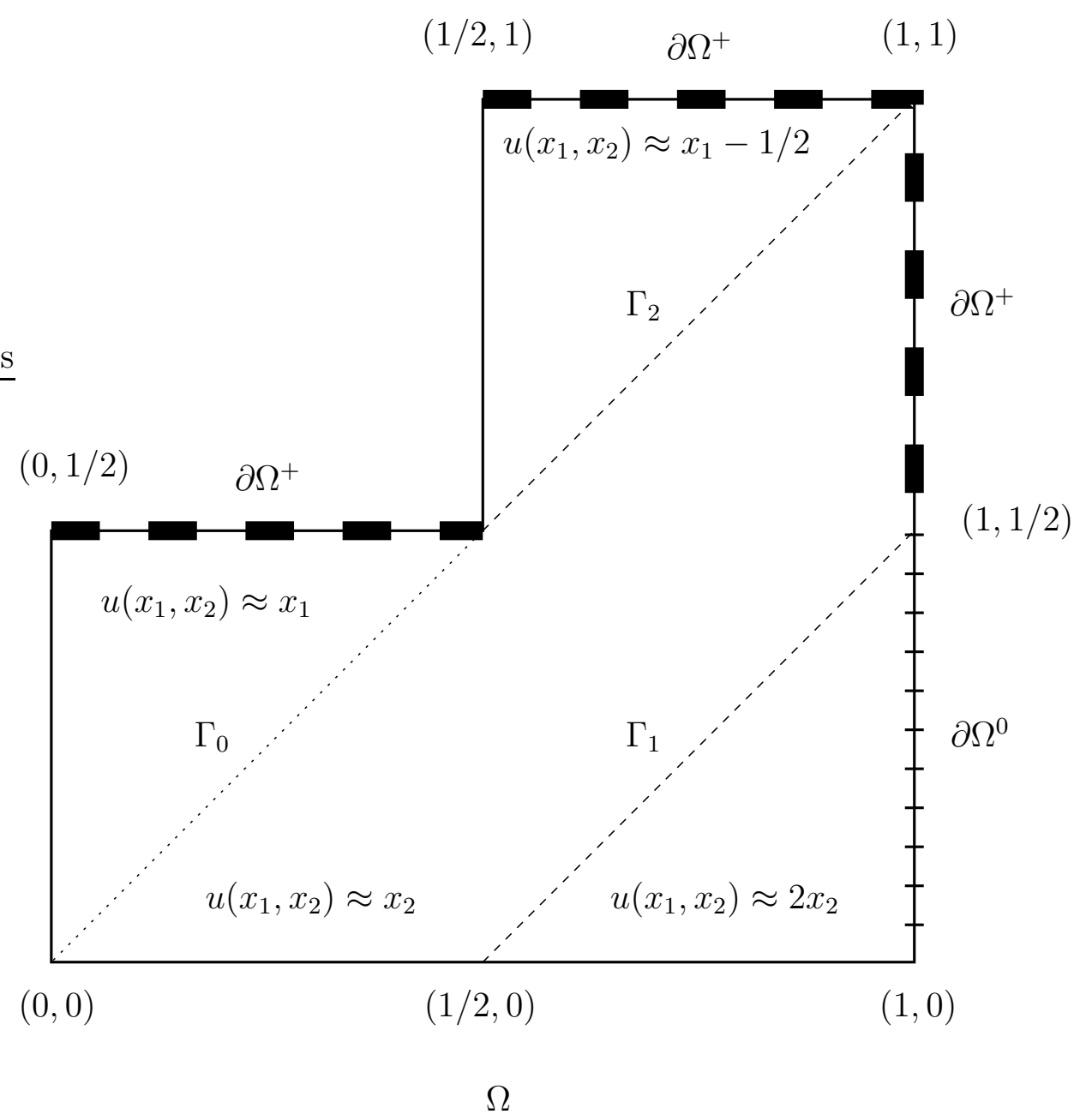

Figure 4: Structure of the exact solution $u$ for the test problem.

$\left\|u-u^{D B}\right\|_{L^{2}}$ ), on the different meshes. It is clear that the presence of spurious oscillations does not affect the order of the methods - the optimal order of convergence in $L^{2}$-norm is $1 / 2$, due to the presence of boundary layers. The discontinuous approximation of the bubbles degrees of freedom $u_{D}^{D B}$ actually improves the accuracy when added to the macro-scales $u_{P}^{D B}$, since we see that DB is $20 \%-25 \%$ better than SC (and SUPG), or, in other words, DB is as accurate as $\mathrm{SC}$ on a two-times finer mesh.

We have evaluated the numerical errors for the previous test-cases in different subregions of the domain. The results, not reported here, shows that the improvement of DB versus $\mathrm{SC}$ is maximal in exponential boundary layers, where actually DB gives a highly discontinuous numerical solution, while the two methods are nearly equivalent on internal layers. This reveals that the bubble part $u_{B}^{R F B}$ of the RFB solution, which we approximate through $u_{D}^{D B}$, produces a proper contribution. 

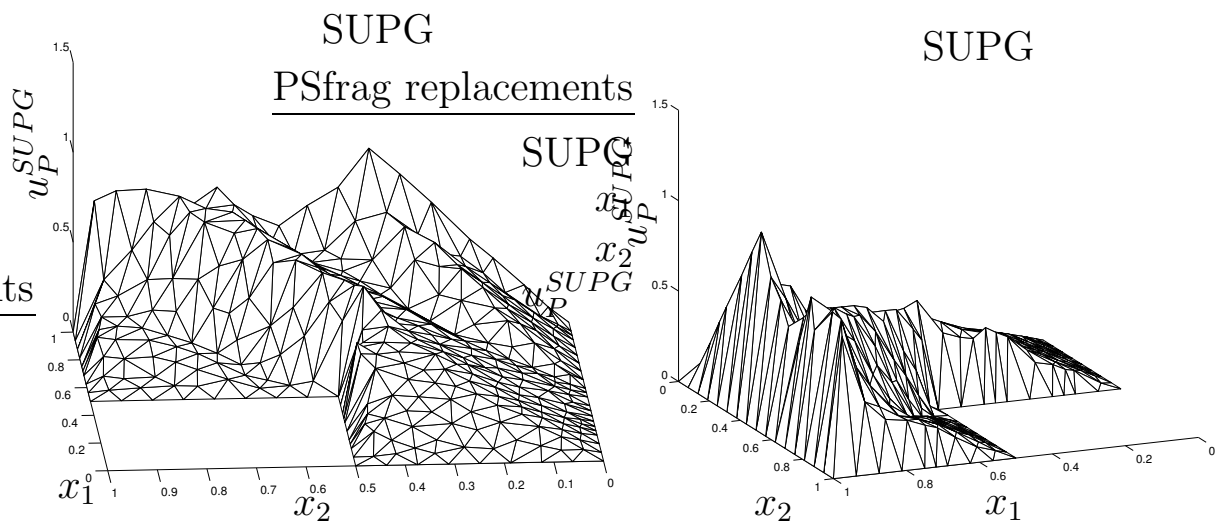

(a)
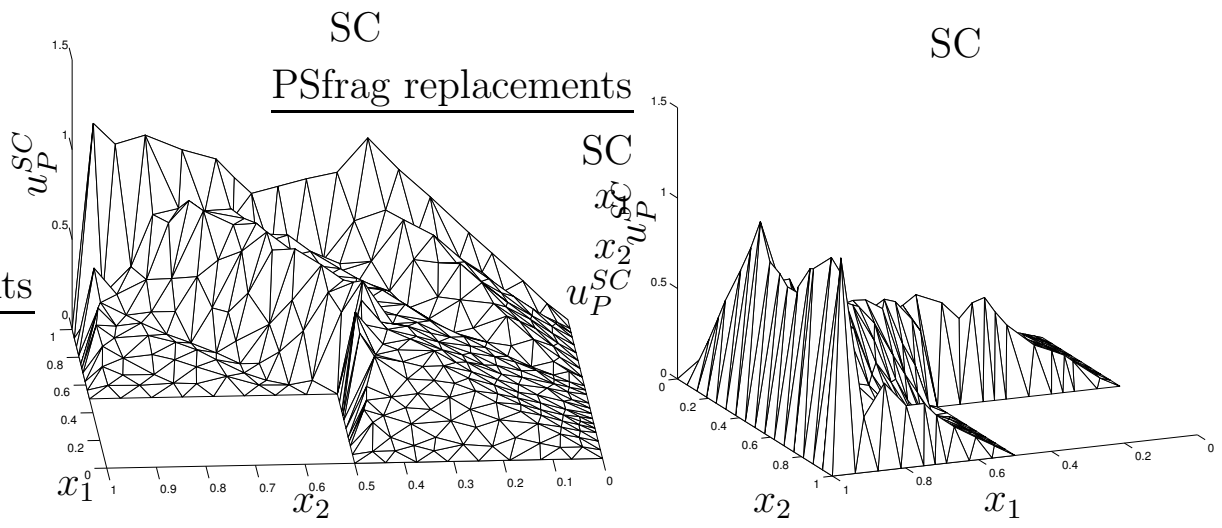

(b)
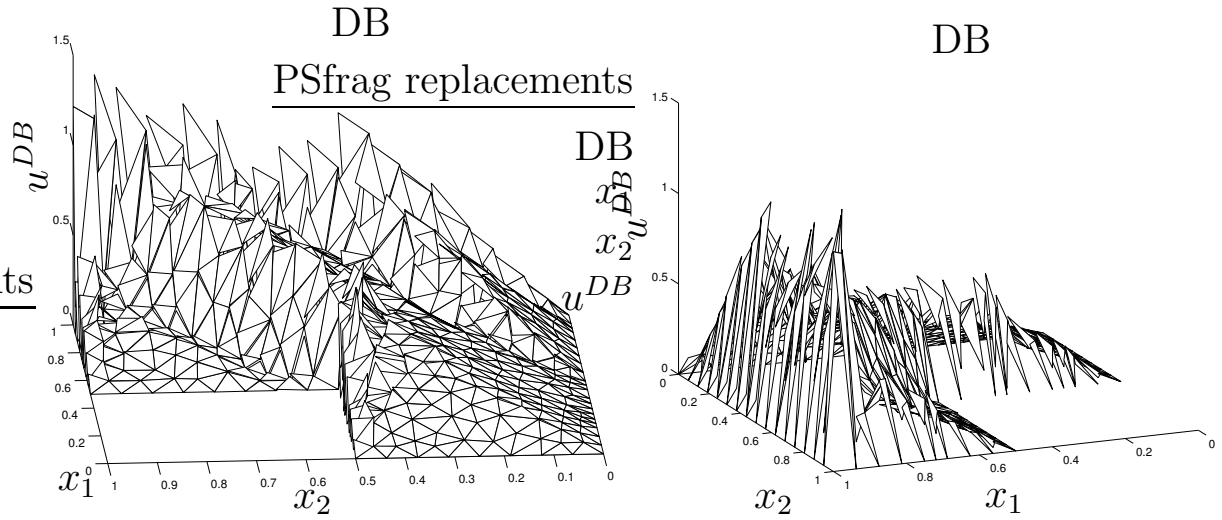

(c)

Figure 5: Plot of $u^{S U P G}(\mathrm{a}), u_{P}^{S C}$ (b), and $u^{D B}$ (c) for the model problem. 


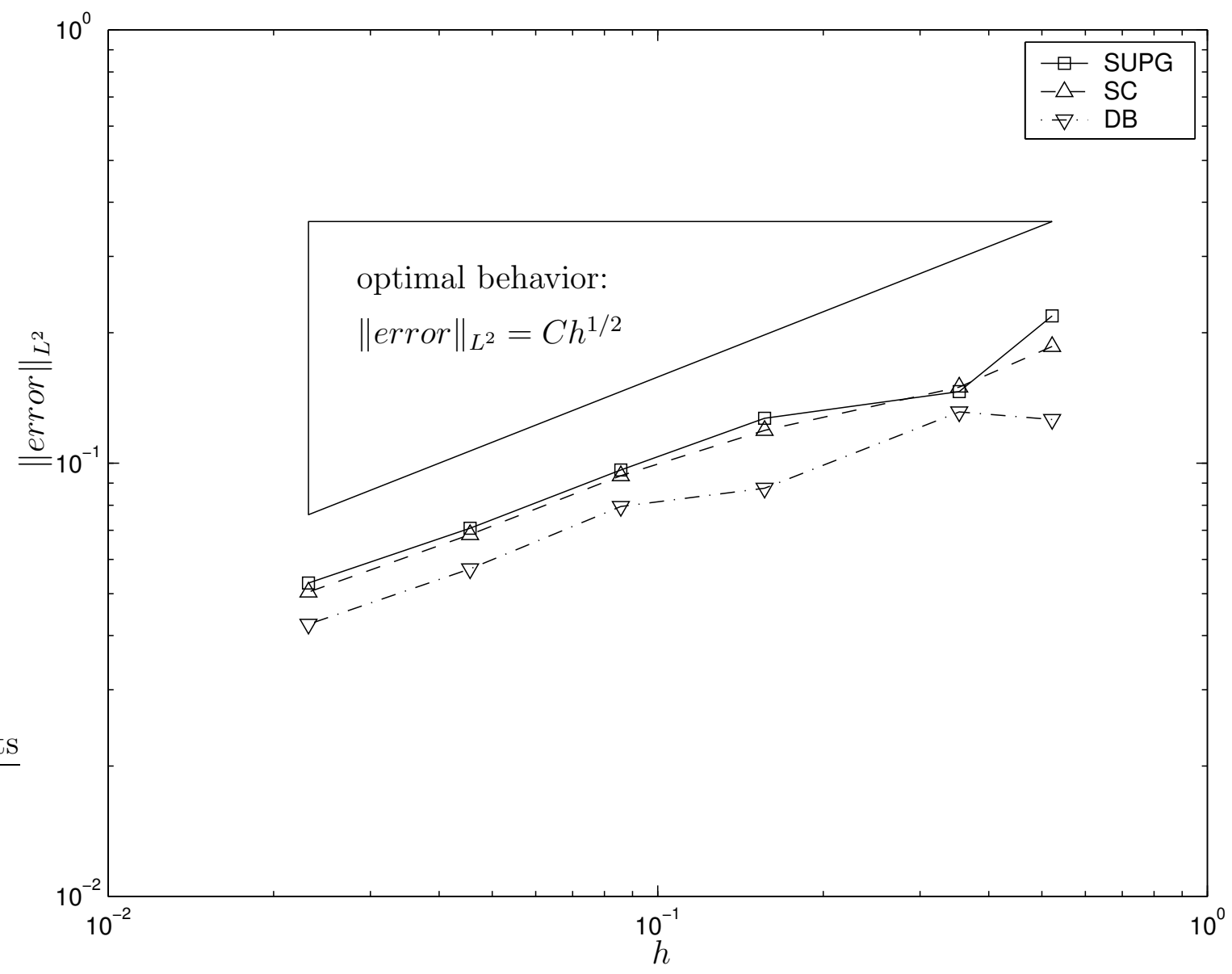

Figure 6: Convergence analysis with respect to the $L^{2}$-norm. 


\section{Conclusions}

In this paper, we have proposed an implementation of the Residual-Free Bubble (RFB) method for the advection-diffusion linear problem in the advection-dominated regime. The ResidualFree Bubble (RFB) method is a general methodology for solving partial differential equations. From an abstract standpoint, it is based on a Finite Element formulation on an enriched space, in which the standard piecewise polynomial functions are enriched by means of bubbles, i.e., functions whose support remains inside the elements. The bubbles make the whole formulation intrinsically stable.

For a practical implementation of the Residual-Free Bubble (RFB) formulation, one has to approximate the infinitely many degrees of freedom of the bubble, to suitably approximate the local problems. We use for that purpose a discontinuous method, which has the advantage of computing in an accurate way the effect of the bubble on the coarse-scale, where the coarse-scale are piecewise linear or quadratic polynomials.

We test the procedure for linear elements at the coarse-scale level. Other studies have been devoted to the numerical testing of Residual-Free Bubble (RFB) based procedures, and we confirm here that the results are favorable in comparison to the popular Streamline-Upwind Petrov-Galerkin (SUPG) numerical results.

Interest in analyzing and developing the Residual-Free Bubble (RFB) methodology, as well as other multiscale methodologies, stems from the realization that these are a quite general methodologies whose validity in other contests has been confirmed in many recent investigations (see, e.g., $[1,2,4,11,13,24]$ ).

\section{References}

[1] Asensio, M., A. Russo, and G. Sangalli: 2003, 'The Residual-Free Bubble numerical method with quadratic elements'. Technical report, I.M.A.T.I.-C.N.R., Pavia. Math. Models Methods Appl. Sci., submitted.

[2] Brezzi, F.: 2000, 'Interacting with the subgrid world'. In: Numerical analysis 1999 (Dundee). Chapman \& Hall/CRC, Boca Raton, FL, pp. 69-82.

[3] Brezzi, F., L. P. Franca, T. J. R. Hughes, and A. Russo: 1997a, 'b= $b$ g'. Comput. Methods Appl. Mech. Engrg. 145(3-4), 329-339.

[4] Brezzi, F., L. P. Franca, T. J. R. Hughes, and A. Russo: 1997b, 'Stabilization techniques and subgrid scales capturing'. In: The state of the art in numerical analysis (York, 1996). New York: Oxford Univ. Press, pp. 391-406.

[5] Brezzi, F., D. Marini, P. Houston, and E. Süli: 2000a, 'Modeling subgrid viscosity for advection-diffusion problems'. Comput. Methods Appl. Mech. Engrg. 190(1-2), 1601-1610.

[6] Brezzi, F., D. Marini, and A. Russo: 1998, 'Applications of the pseudo residual-free bubbles to the stabilization of convection-diffusion problems'. Comput. Methods Appl. Mech. Engrg. 166(1-2), 51-63.

[7] Brezzi, F., D. Marini, and E. Süli: 2000b, 'Residual-free bubbles for advection-diffusion problems: the general error analysis'. Numer. Math. 85(1), 31-47. 
[8] Brezzi, F., D. Marini, and E. Süli: 2000c, 'Residual-free bubbles for advection-diffusion problems: the general error analysis'. Numer. Math. 85(1), 31-47.

[9] Brezzi, F. and L. D. Marini: 2002, 'Augmented spaces, two-level methods, and stabilizing subgrids'. Internat. J. Numer. Methods Fluids 40(1-2), 31-46. ICFD Conference on Numerical Methods for Fluid Dynamics (Oxford, 2001).

[10] Brezzi, F. and A. Russo: 1994, 'Choosing bubbles for advection-diffusion problems'. Math. Models Methods Appl. Sci. 4(4), 571-587.

[11] Brezzi, F. and A. Russo: 2000, 'Stabilization techniques for the finite element method'. In: Applied and industrial mathematics, Venice-2, 1998. Dordrecht: Kluwer Acad. Publ., pp. $47-58$.

[12] Brooks, A. N. and T. J. R. Hughes: 1982, 'Streamline upwind/Petrov-Galerkin formulations for convection dominated flows with particular emphasis on the incompressible Navier-Stokes equations'. Comput. Methods Appl. Mech. Engrg. 32(1-3), 199-259. FENOMECH '81, Part I (Stuttgart, 1981).

[13] Canuto, C., A. Russo, and V. van Kemenade: 1998, 'Stabilized spectral methods for the Navier-Stokes equations: residual-free bubbles and preconditioning'. Comput. Methods Appl. Mech. Engrg. 166(1-2), 65-83.

[14] Ciarlet, P. G.: 2002, The finite element method for elliptic problems, Vol. 40 of Classics in Applied Mathematics. Philadelphia, PA: Society for Industrial and Applied Mathematics (SIAM). Reprint of the 1978 original [North-Holland, Amsterdam; MR 58 \#25001].

[15] Franca, L. P., A. Nesliturk, and M. Stynes: 1998, 'On the stability of residual-free bubbles for convection-diffusion problems and their approximation by a two-level finite element method'. Comput. Methods Appl. Mech. Engrg. 166(1-2), 35-49.

[16] Guermond, J.-L.: 1999, 'Stabilization of Galerkin approximations of transport equations by subgrid modeling'. M2AN Math. Model. Numer. Anal. 33(6), 1293-1316.

[17] Hughes, T. J. R., G. R. Feijóo, L. Mazzei, and J.-B. Quincy: 1998, 'The variational multiscale method - a paradigm for computational mechanics'. Comput. Methods Appl. Mech. Engrg. 166(1-2), 3-24.

[18] Hughes, T. J. R., L. P. Franca, and G. M. Hulbert: 1989, 'A new finite element formulation for computational fluid dynamics. VIII. The Galerkin/least-squares method for advectivediffusive equations'. Comput. Methods Appl. Mech. Engrg. 73(2), 173-189.

[19] Hughes, T. J. R. and M. Mallet: 1986, 'A new finite element formulation for computational fluid dynamics. III. The generalized streamline operator for multidimensional advectivediffusive systems'. Comput. Methods Appl. Mech. Engrg. 58(3), 305-328.

[20] Lions, J.-L. and E. Magenes: 1973, Non-homogeneous boundary value problems and applications. Vol. III. New York: Springer-Verlag. Translated from the French by P. Kenneth, Die Grundlehren der mathematischen Wissenschaften, Band 183. 
[21] Quarteroni, A. and A. Valli: 1994, Numerical approximation of partial differential equations. Berlin: Springer-Verlag.

[22] Roos, H.-G., M. Stynes, and L. Tobiska: 1996, Numerical methods for singularly perturbed differential equations. Berlin: Springer-Verlag. Convection-diffusion and flow problems.

[23] Sangalli, G.: 2000, 'Global and local error analysis for the residual-free bubbles method applied to advection-dominated problems'. SIAM J. Numer. Anal. 38(5), 1496-1522 (electronic).

[24] Sangalli, G.: 2003, 'Capturing Small Scales in Elliptic Problems Using a Residual-Free Bubbles Finite Element Method'. Multiscale Modeling and Simulation 1(3), 485-503.

[25] Shewchuk, J. R.: 1996, 'Triangle: Engineering a 2D Quality Mesh Generator and Delaunay Triangulator'. In: M. C. Lin and D. Manocha (eds.): Applied Computational Geometry: Towards Geometric Engineering, Vol. 1148 of Lecture Notes in Computer Science. SpringerVerlag, pp. 203-222. From the First ACM Workshop on Applied Computational Geometry. 